\section{Aspectos epidemiológicos da hanseníase: uma abordagem espacial}

\author{
Epidemiological aspects of leprosy: a spatial \\ approach
}

\author{
${ }_{1}$ Departamento de Vigilância \\ em Saúde, Secretaria \\ Municipal de Saúde de \\ Duque de Caxias, Duque de \\ Caxias, Brasil. \\ 2 Escola Nacional de Saúde \\ Pública Sergio Arouca, \\ Fundação Oswaldo Cruz, Rio \\ de Janeiro, Brasil. \\ 3 Faculdade de Ciências \\ Médicas, Universidade do \\ Estado do Rio de Janeiro, Rio \\ de Janeiro, Brasil. \\ 4 Hospital Universitário \\ Clementino Fraga Filho, \\ Universidade Federal do Rio \\ de Janeiro, Rio de Janeiro, \\ Brasil. \\ Correspondência \\ M. Duarte-Cunha \\ Departamento de Vigilância \\ em Saúde, Secretaria \\ Municipal de Saúde de \\ Duque de Caxias. \\ Rua James Franco 3, Duque \\ de Caxias, $R J$ \\ 25215-260, Brasil. \\ monicadacunha@gmail.com
}

,

\section{Abstract}

The objective of this study was to identify spatial patterns in the occurrence of leprosy in Duque de Caxias, a municipality (county) with high endemicity for the disease in the State of Rio de Janeiro, Brazil. The authors selected all new leprosy cases reported to the Brazilian National Database on Diseases of Notification (SINAN) from 1998 to 2006. The analysis was performed according to three-year periods, followed by spatial analysis according to the local empirical Bayesian method and calculation of global (Moran) and local (LISA) spatial autocorrelation. The analysis showed marked improvement in the epidemiological situation associated with earlier diagnosis. There was a reduction in the proportion of cases with grade II leprosy, from $13.6 \%$ to 8.6\% ( $p=0.04)$, and increased detection of cases with the indeterminate form, from $10.3 \%$ to $18 \%$ $(p=0.00)$. Spatial analysis identified a cluster in the South-Northwest of the municipality, not directly related to the actions or decentralization campaign, proving to be an important tool for identifying critical areas for the endemic and assessing the impact of strategic measures to combat the disease.

Leprosy; Spatial Analysis; Endemic Diseases
Mônica Duarte-Cunha 1 Reinaldo Souza-Santos 2 Haroldo José de Matos 3 Maria Leide W. de Oliveira 4

\section{Introdução}

A eliminação da hanseníase tem sido difícil de ser alcançada em algumas regiões, em face da complexidade da doença 1,2. Sabe-se que o Mycobacterium leprae é considerado de baixa patogenicidade, pois apenas uma pequena parcela dos indivíduos supostamente infectados desenvolve a moléstia ${ }^{3}$. Além disso, não se sabe ao certo se, em algum momento entre o contato com o bacilo e a enfermidade, o indivíduo assintomático se torna "bacilífero" e participa da cadeia de transmissão da hanseníase ${ }^{4}$. Esclarecer que a infecção em indivíduos assintomáticos pode disseminar a doença é vital à formulação de ações estratégicas ao manejo do problema 5,6.

Por que, em algumas áreas, a hanseníase torna-se endêmica, apesar de ações intensas de combate? Existiriam fatores geográficos, socioculturais ou de dinâmica e fluxo econômicos e de ocupação influenciando a circulação permanente do bacilo na população? A proximidade das pessoas que vivem em áreas de alta densidade populacional poderia ser um fator agravante do risco 7 ? Infecção subclínica seria uma fonte de transmissão oculta, funcionando como um perpetuador da endemia $5,8,9$ ?

Diante do não conhecimento de todos os aspectos clínicos e laboratoriais da hanseníase, como a inexistência de um modelo experimental sólido e a impossibilidade de se cultivar o M. leprae, torna-se difícil a compreensão de pon- 
tos cruciais da enfermidade, como a transmissão, o neurotropismo da micobactéria e a suscetibilidade de alguns indivíduos para o desenvolvimento de formas mais graves ou mais bacilíferas 2 . Portanto, o estudo epidemiológico da hanseníase permanece, ainda hoje, como uma ferramenta poderosa para a compreensão do seu ciclo de transmissão.

No Município de Duque de Caxias, Rio de Janeiro, ao longo do período do estudo, foram realizadas, entre outras, duas importantes estratégias operacionais no combate à doença. A primeira foi a descentralização da assistência no diagnóstico e tratamento da hanseníase, seguindo recomendação da Organização Mundial da Saúde (OMS) e do Ministério da Saúde, a fim de atingir a meta de menos de um caso a cada 10 mil habitantes, no que se refere à eliminação da doença como problema de saúde pública 10,11. Essa descentralização iniciou-se, em 1998, nas unidades de referência com dermatologista (Posto Médico Sanitário - PMS) e se expandiu progressivamente para as unidades básicas (unidades do Programa Saúde da Família - PSF), facilitando a detecção precoce de casos e a aderência do paciente ao tratamento 12,13. A segunda estratégia foi a realização de várias campanhas focalizadas em pequenas áreas e envolvendo unidades de saúde, em parceria com o serviço de dermatologia da Universidade Federal do Rio de Janeiro (UFRJ). Essa parceria iniciou em 2002 através de projeto de pesquisa da UFRJ e, portanto, até então o município realizava apenas campanhas anuais associadas à campanha de vacinação contra poliomielite que ocorre rotineiramente no país. O objetivo foi um rastreamento ativo de casos novos e divulgação sobre a doença à população. Em adição, a campanha foi uma ferramenta facilitadora e de apoio para a descentralização das unidades participantes 13,14.

Assim como em todo o Brasil, a implantação do banco Sistema Nacional de Agravos de Notificações (SINAN) foi iniciada no Município de Duque de Caxias na primeira metade da década de 1990, com a utilização do aplicativo Sinan-DOS a partir de 1993. A regulamentação por decreto ministerial só ocorreu em 1998 15; naquela época, o banco municipal já apresentava boa qualidade e confiabilidade no que tange à baixa taxa de subnotificações.

O quanto a estratégia de controle da transmissão da hanseníase é capaz de prevenir e controlar a doença precisa ser analisado de forma crítica. A inclusão da dimensão geográfica no rol das possibilidades de análise dos dados de vigilância epidemiológica busca ampliar o seu poder explicativo acerca dos processos de produção dos agravos de interesse para a saúde pública, uma vez que leva em conta os diferentes padrões de acesso aos bens e serviços urbanos, evidenciando, dessa forma, as desigualdades existentes no interior dos municípios. As técnicas de análise espacial podem ser uma nova forma de avaliar o contexto e seus fatores de risco 16, aperfeiçoando o planejamento de intervenções e monitoramentos seletivos conforme as reais necessidades de pequenas áreas 17 .

O objetivo deste trabalho foi identificar o padrão espacial da ocorrência da hanseníase em Duque de Caxias, município de alta endemicidade da doença no Estado do Rio de Janeiro, sendo considerada área prioritária para o Ministério da Saúde no combate à hanseníase.

\section{Material e métodos}

Trata-se de um estudo ecológico, tendo como unidade de análise os bairros do Município de Duque de Caxias, situado na Região Metropolitana do Estado do Rio de Janeiro, Região Sudeste do Brasil. A cidade tem $442 \mathrm{~km}^{2}$ de extensão territorial e está localizada a $22^{\circ} 47^{\prime} 10^{\prime \prime}$ de latitude Sul e 4318'30" de longitude Oeste do meridiano de Greenwich, compondo a região da Baixada Fluminense. Seu território está subdividido em quatro áreas distritais, sendo o quarto distrito o que tem maior extensão territorial, correspondendo a $239 \mathrm{~km}^{2}$ (54\% de todo o município). Conta com população estimada, em 2004, de 830.679 habitantes e proporção, por gênero, de $48 \%$ de homens. Em todo o município, 31\% das residências não têm abastecimento de água potável, e somente $56,4 \%$ utilizam sistema de esgoto sanitário. Aproximadamente 99\% dos indivíduos vivem em área urbana, sendo $78 \%$ localizadas no primeiro e segundo distritos. $\mathrm{O}$ terceiro distrito apresenta aspecto de transição urbano-rural, e o quarto distrito, predominantemente rural. Em 2002, a taxa de mortalidade infantil era de 18,7/ mil nascidos vivos, com redução de $45,3 \%$ no período de 1993 a 2002. No mesmo ano, a taxa de alfabetização na população de 10 anos ou mais de idade foi de 92,4\% (Instituto Brasileiro de Geografia e Estatística. Cidades@.http://www.ibge. gov.br/cidade@, acessado em 20/Dez/2004).

Utilizaram-se os dados referentes aos casos novos de hanseníase residentes no Município de Duque de Caxias, registrados entre 1998 e 2006, no banco SINAN municipal. Optou-se por esse período em virtude de esses dados se apresentarem com melhor qualidade e confiabilidade, principalmente aqueles relacionados às baixas subnotificações e à alta proporção de identificação do bairro de residência do caso. A partir de 
2003, a Prefeitura Municipal de Duque de Caxias promoveu uma reformulação dos bairros, agregando os pequenos a outros maiores, reduzindo de 426 para 40 bairros oficiais. Para este estudo, todos os bairros anteriores a 2003 foram reclassificados, considerando-se apenas os 40 bairros da classificação atual.

Os dados foram subdivididos em três períodos: P1 (1998 a 2000), P2 (2001 a 2003) e P3 (2004 a 2006), sendo também utilizado o período total T (1998 a 2006). Essa subdivisão teve como intenção captar melhor as oscilações dos dados, uma vez que nas doenças crônicas essas oscilações são mais lentas e difíceis de serem percebidas. A malha digital do município foi obtida no site do Instituto Brasileiro de Geografia e Estatística (IBGE; http://www.ibge.gov.br), assim como a população de cada bairro, a partir dos setores censitários do Censo Demográfico de 2000, por meio de operações entre camadas em Sistema de Informação Geográfica.

As seguintes covariáveis foram utilizadas: número de casos novos, sexo, faixa etária (0-14 anos, 15-59 anos e 60 anos ou mais), classificação operacional (multibacilar - MB, paucibacilar - PB), forma clínica (indeterminada - I, tuberculóide $-\mathrm{T}$, dimorfa $-\mathrm{D}$, virchoviana $-\mathrm{V}$ ), grau de incapacidade física no diagnóstico (0, I e II), número de casos com grau de incapacidade física avaliado no diagnóstico, número de contatos registrados, número de unidades básicas descentralizadas (PSF), número de unidades de referência descentralizadas (PMS) e número de campanhas focalizadas de rastreamento realizadas. Essas informações foram obtidas na Secretaria Municipal de Saúde de Duque de Caxias.

Inicialmente, realizou-se análise dos dados para conhecer o perfil epidemiológico da região. A frequência de casos novos de cada período foi calculada considerando a soma anual para cada bairro. Foram também calculadas as taxas de detecção para cada 10 mil habitantes, para cada período (P1, P2, P3 e T), e construída tabela de contingência incluindo as covariáveis, com testes de significância qui-quadrado de $5 \%$. Os coeficientes de correlação e gráficos de dispersão foram realizados entre as seguintes variáveis, considerando os bairros: (1) frequência de casos novos em $\mathrm{P} 1$ versus $\mathrm{P} 2$, em $\mathrm{P} 1$ versus $\mathrm{P} 3$ e em $\mathrm{P} 2$ versus P3; (2) frequência de casos novos e de campanhas focais, para cada período; (3) frequência de casos novos e de unidade PMS descentralizadas, para cada período; (4) frequência de casos novos e de unidade de PSF descentralizadas, para cada período.

A análise espacial foi feita a partir do banco de dados dos casos novos e da malha municipal, considerando os eventos com representação por área. Foi realizada a construção e análise de mapa temático de cada período (P1, P2, P3 e T), com base na distribuição dos casos de hanseníase observados e das taxas de detecção da doença, em cada bairro de Duque de Caxias. O objetivo foi identificar a existência de algum padrão espacial diferente do aleatório e possível padrão de associação com fatores de risco ${ }^{18}$. A inclusão de informações nos mapas temáticos, referentes à descentralização e campanhas, foi feita definindo-se os extratos a partir do quartil de cada período, padronizado pelo período total. O intervalo dos extratos foi definido pelo P1.

Para possibilitar a análise espacial e a construção dos mapas temáticos, foi necessária a exclusão do polígono ao norte do mapa municipal, correspondente à área de mata de preservação ambiental, que não possui habitantes. Por causa das áreas com populações muito pequenas, utilizou-se o estimador bayesiano empírico para minimizar as flutuações dos dados, tanto para a frequência quanto para as taxas. Utilizou-se a média dos bairros vizinhos (método bayesiano empírico local - LEB) no fator de ponderação desse estimador. A matriz de vizinhança escolhida foi a de contiguidade.

Posteriormente, foi usada a função de autocorrelação espacial para análise da dependência espacial entre as taxas brutas de casos novos nos vários bairros, em cada período. Foi feito teste de significância a partir dos valores da função de autocorrelação de primeira ordem global (Moran) e local (LISA), para verificar a presença de cluster espacial ou "bolsões" de não estacionariedade, que são áreas com dinâmica espacial própria, em toda a área de estudo e entre vizinhos, respectivamente. Foi considerado o teste de significância de $1 \%$. Com base na análise da autocorrelação local, foram construídos mapas, denominados "LISA MAP”, indicativos das regiões que apresentam correlação local significativamente diferente do observado no resto dos dados 19 .

Para a identificação das áreas de maior, menor e intermediária detecção de casos de hanseníase, elaboraram-se mapas a partir dos resultados do índice de Moran local (BoxMap) das taxas brutas e ajustadas (LEB), considerando-se os bairros agregados conforme os quadrantes do diagrama de espalhamento de Moran. Para Q1 (+/+), bairros com alta taxa detecção e vizinhos também com alta taxa de detecção; Q2 (-/-), bairros com baixa taxa detecção e vizinhos também com baixa taxa de detecção; Q3 (+/-), bairros com alta taxa detecção e vizinhos com baixa taxa de detecção; Q4 (-/+), bairros com baixa taxa detecção e vizinhos com alta taxa de detecção. Em Q1 e Q2, a associação espacial é positiva (bairros e seus vizinhos com valores semelhantes); por outro 
lado, em Q3 e Q4, a associação espacial é negativa (bairros e seus vizinhos com valores diferentes).

A construção das taxas foi desenvolvida no programa Excel 2003, enquanto os gráficos de dispersão e cálculo do coeficiente de correlação foram realizados no programa SPSS versão 12.0 (SPSS Inc., Chicago, Estados Unidos). Os mapas, assim como a análise de autocorrelação, foram obtidos no programa TerraView versão 3.2.1 (Instituto Nacional de Pesquisas Espaciais; http:// www.dpi.inpe.br/terraview).

\section{Resultados}

\section{Análise descritiva}

A análise inicial dos dados permitiu, de forma geral, descrever o perfil da doença em Duque de Caxias, no período estudado. Os dados mostram que o município passou por mudanças acentuadas entre 1998 e 2006, relacionadas, principalmente, à detecção precoce dos casos. Na Tabela 1 , observa-se um pequeno aumento do número de casos novos de P1 (867) para P2 (980) e posterior redução para P3 (725). O mesmo é verificado nas taxas de detecção, variando de 3,88 em P1, para 4,10 em P2, e desse para 3,90 em P3.
A detecção de casos novos em menores de 15 anos sofreu um aumento progressivo, de 5,8\%, em P1, para 8,4\%, em P3, porém sem significância estatística (valor de $\mathrm{p}=0,0968$ ). Já suas taxas mostram evolução com pouca oscilação (de 0,77 em P1 para 0,82 em P3). Quanto aos casos novos detectados em mulheres, verifica-se aumento na detecção de P1 a P2 (52,8\% e 55,1\%, respectivamente) e posterior redução de P2 a P3 $(55,1 \%$ e $49,5 \%$, respectivamente), com valor de p não significativo.

Variáveis como forma clínica e grau de incapacidade podem descrever a precocidade do diagnóstico. É possível observar, em todo o período, uma redução da proporção de casos com grau II de incapacidade física (forma tardia) em relação aos graus 0 e I. Em P1, essa proporção foi de 13,58\% e, em P3, 8,58\%, com valor de $p$ de 0,04 . Em contrapartida, verifica-se aumento da detecção de casos com forma indeterminada (forma precoce) de P1 (10,28\%) para P2 (25,86\%) e redução de P2 para P3 (17,97\%), embora um aumento tenha sido mantido em todo o período, com um pico importante em P2 (valor de $\mathrm{p}=0,0001)$.

A proporção de casos novos com zero contato registrado é uma variável que mede qualidade de serviço, refletindo falha no registro dos contatos.

Hanseníase no Município de Duque de Caxias, Estado do Rio de Janeiro, Brasil, segundo covariáveis individuais e clínicas, 1998 a 2006.

\begin{tabular}{|c|c|c|c|c|c|c|c|c|c|}
\hline \multirow[t]{2}{*}{ Variáveis } & \multicolumn{2}{|c|}{ 1998-2000 (P1) } & \multicolumn{2}{|c|}{ 2001-2003 (P2) } & \multicolumn{2}{|c|}{ 2004-2006 (P3) } & \multicolumn{3}{|c|}{$1998-2006(T)$} \\
\hline & n & $\%$ & $\mathrm{n}$ & $\%$ & $\mathrm{n}$ & $\%$ & $\mathrm{n}$ & $\%$ & Qui-quadrado \\
\hline Casos novos & 867 & 33,70 & 980 & 38,10 & 725 & 28,20 & 2.572 & - & - \\
\hline Casos novos em menores de 15 anos & 50 & 5,80 & 63 & 6,40 & 61 & 8,40 & 174 & 6,77 & 0,0968 \\
\hline Casos novos em mulheres & 458 & 52,80 & 540 & 55,10 & 359 & 49,50 & 1.357 & 52,80 & 0,0736 \\
\hline $\begin{array}{l}\text { Casos novos com grau Il de incapacidade } \\
\text { física/Casos novos com grau } 0 \text { e I de } \\
\text { incapacidade física }\end{array}$ & 0,14 & 13,59 & 0,10 & 9,91 & 0,09 & 8,58 & 0,11 & 10,88 & 0,0400 \\
\hline $\begin{array}{l}\text { Casos novos com grau de incapacidade física } \\
\text { realizado }\end{array}$ & 719 & 83,00 & 632 & 67,10 & 544 & 79,20 & 1.895 & 76,00 & 0,0000 \\
\hline Multibacilar/Paucibacilar & 1,06 & - & 1,01 & - & 1,01 & - & 1,03 & - & 0,0893 \\
\hline $\begin{array}{l}\text { Forma clínica indeterminada/Forma } \\
\text { tuberculoide, dimorfa e virchowiana }\end{array}$ & 0,10 & 10,28 & 0,26 & 25,86 & 0,18 & 17,97 & 0,18 & 18,01 & 0,0000 \\
\hline Casos novos sem contatos registrados & 172 & 19,80 & 404 & 45,00 & 2 & 0,40 & 578 & 25,70 & 0,0000 \\
\hline Média de contatos de casos novos registrados & 5,67 & - & 5,24 & - & 4,67 & - & 5,26 & - & - \\
\hline $\begin{array}{l}\text { Taxa de detecção de casos novos (por } 10.000 \\
\text { habitantes) }\end{array}$ & 3,88 & - & 4,10 & - & 3,90 & - & 3,61 & - & - \\
\hline $\begin{array}{l}\text { Taxa de detecção em menores de } 15 \text { anos } \\
\text { (por } 10.000 \text { habitantes) }\end{array}$ & 0,77 & - & 0,87 & - & 0,82 & - & 0,84 & - & - \\
\hline
\end{tabular}


Observa-se que houve uma redução significante dos valores dessa variável em todo o período (de 19,8 em P1 para 0,4\% em P3).

Ao avaliar a existência de associação do número de casos novos detectados entre os períodos, verifica-se, nos gráficos de dispersão, correlação expressiva e significante estatisticamente, com $r=0,77$ entre P1 e P2; $r=0,80$ entre P1 e P3; $r=0,92$ entre P2 e P3. Destacam-se alguns bairros com aumento significativo, a saber: Gramacho, Imbariê, Parque Paulista, Santa Lúcia, São Bento e Chácara Arcampo entre P1 e P2; Gramacho, Pilar, Parque Fluminense, Imbariê, Parque Paulista, Xerém e Parada Angélica entre P1 e P3; Pilar, Parque Fluminense, Bar dos Cavaleiros, Campos Elíseos e Parada Angélica entre P2 e P3. Os três gráficos mostram associação semelhante, sendo ilustrado na Figura 1 apenas o gráfico de dispersão entre o primeiro e último período ( $\mathrm{P} 1$ versus $\mathrm{P} 3$ ).

A análise da associação entre os casos novos de todo o período (1998 a 2006) e as campanhas focalizadas (Figura 1) evidenciou uma correlação positiva, com $r=0,55$ significante (valor de $\mathrm{p}=0,0001$ ). Contudo, em três bairros (Bar dos Cavaleiros, Vila São Luís e Santa Cruz da Serra), apesar de não terem sido realizadas campanhas, houve maior detecção de casos novos.

Não foi verificada correlação do número de casos novos com o número de unidades descentralizadas de atendimento a hanseníase, tanto de PSF como de PMS, para todos os períodos.

\section{Análise espacial}

O resultado da descrição do perfil da população do município foi construído mediante mapa temático, ilustrando a densidade populacional em cada bairro do município (Figura 2). A área em branco corresponde a reservas florestais importantes da Mata Atlântica. Observa-se um gradiente crescente da densidade no sentido norte-sul.

A análise dos mapas com a distribuição espacial do número de casos novos para cada período (Figura 3) evidencia, em todos os períodos, que há aumento da detecção de casos nos bairros onde se realizaram mais ações estratégicas, formando uma faixa do sul até o nordeste do município. Os bairros onde se detectaram mais casos em todo o período foram Saracuruna (36), Jardim Primavera (19), Imbariê (17) e Gramacho (16).

Figura 1

Casos de hanseníase por bairro do Município de Duque de Caxias, Rio de Janeiro, Brasil.

a) Casos novos de $1998-2000$ versus casos novos de 2004-2006

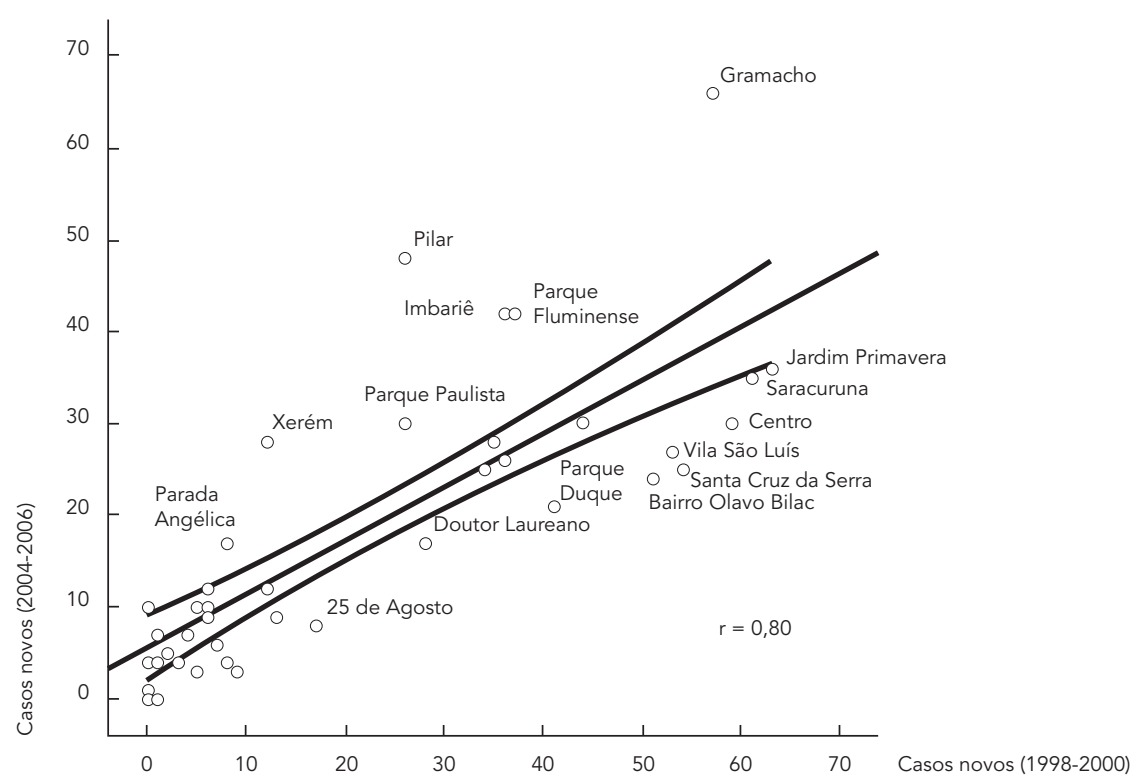

(continua) 
b) Campanhas de rastreamento de 1998-2006 versus número de casos novos de 1998-2006

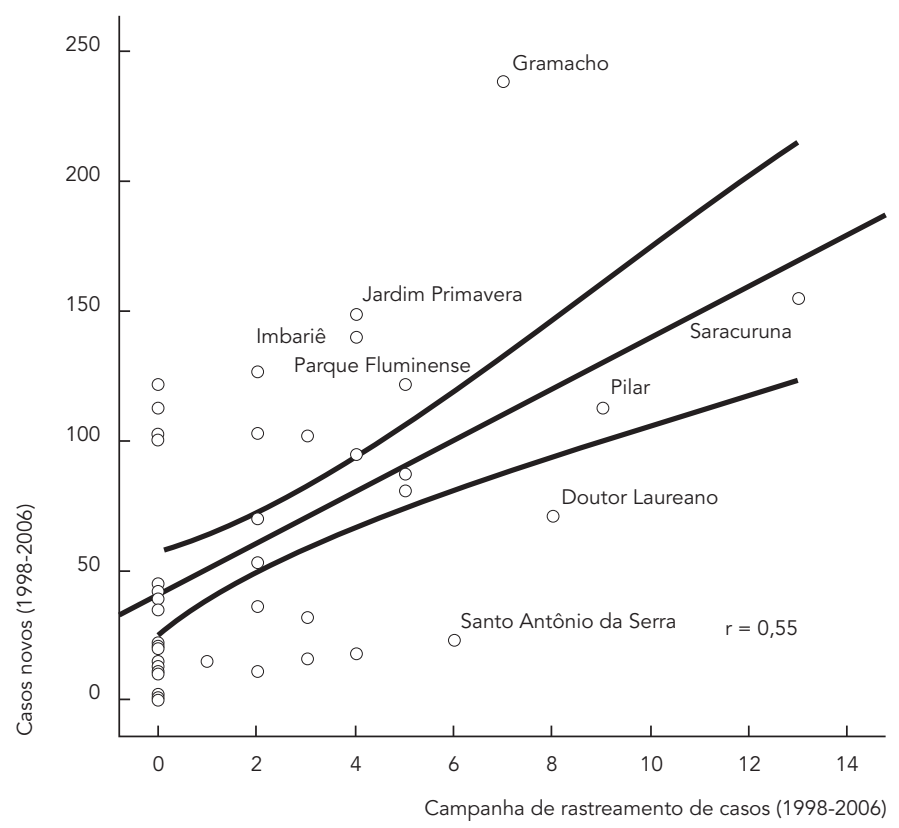

Uma mudança no padrão espacial é observada quando se considera na análise a taxa de detecção bruta. Ao contrário do que ocorreu com a frequência dos casos, as taxas mais altas não estão associadas espacialmente com as estratégias de campanha e descentralização de unidades de atendimento. Isso foi verificado nos quatro períodos com padrões semelhantes, sendo ilustrado na Figura 4 apenas o período T (1998 a 2006).

Há predomínio de bairros com taxas mais altas, ocupando uma faixa, não contínua, que se estende do sul ao noroeste do município, em todos os períodos. Entre eles estão: Parque Capivari, Centenário, Centro, Cidade dos Meninos, Pilar e Parque Sarapuí.

Quando se analisam os mapas com as taxas ajustadas pelo LEB, a faixa do sul ao noroeste do município se mantém de forma semelhante (Figura 4).

Os resultados da análise do índice de Moran global para a taxa bruta dos casos novos, no P1 $(-0,003$; valor de $\mathrm{p}=0,38)$, no $\mathrm{P} 2(-0,05$; valor de $\mathrm{p}=0,18)$, no P3 $(0,10$; valor de $\mathrm{p}=0,09)$ e T $(-0,04$; valor de $\mathrm{p}=0,26$ ), não mostraram presença de cluster espacial (não estacionariedade) em toda a área do município.
A análise de autocorrelação local, por meio do LISA MAP e do BoxMap, das taxas brutas e ajustadas, apenas do período T, está ilustrada na Figura 5. Os resultados de P1, P2 e P3 não mostraram alterações relevantes que fossem diferentes daquelas visualizadas no período T. No LISA MAP, não foi verificada uma diferença na distribuição espacial dos bairros que tiveram correlação estatisticamente significante, entre as taxas brutas e ajustadas. Para as taxas ajustadas, há maior agregação a noroeste do município. Alguns bairros do município ao norte (Mantiqueira e Santa Cruz da Serra) e sul (Doutor Lauriano, Vila São Luís e Parque Duque), mostraram significância para ambas as taxas. Quanto ao BoxMap, é possível observar uma faixa de agregação dos bairros do quadrante Q1, semelhante àquela visualizada nos mapas de distribuição das taxas brutas e ajustadas (Figuras 4), confirmando estatisticamente a faixa.

\section{Discussão e conclusão}

Numa visão mais geral mostrada pela análise dos dados descritivos do município, verificou-se uma 
1 Reserva

2 Bairro Amapá

3 Bar dos Cavalheiros

4 Barro Branco

5 Campos Elíseos

6 Cangulo

7 Parque Capivari

8 Centenário

9 Centro

10 Chácara Arcampo

11 Chácaras Rio-Petrópolis

12 Cidade dos Meninos

13 Parque Paulista

14 Doutor Laureano

15 Figueira

16 Gramacho

17 Imbariê

18 Jardim Anhangá

19 Jardim Primavera

20 Lamarão

21 Mantiquira

22 Meio da Serra

23 Bairro Olavo Bilac

24 Parada Angélica

25 Parada Morabi

26 Parque Duque

27 Eldorado

28 Parque Fluminense

29 Parque Sarapuí

30 Periquitos

31 Pilar

32 Santa Cruz da Serra

33 Santa Lúcia

34 Santo Antônio da Serra

35 São Bento

36 Saracuruna

37 Taquara

38 Vila São José

39 Vila São Luís

4025 de Agosto

41 Xerém

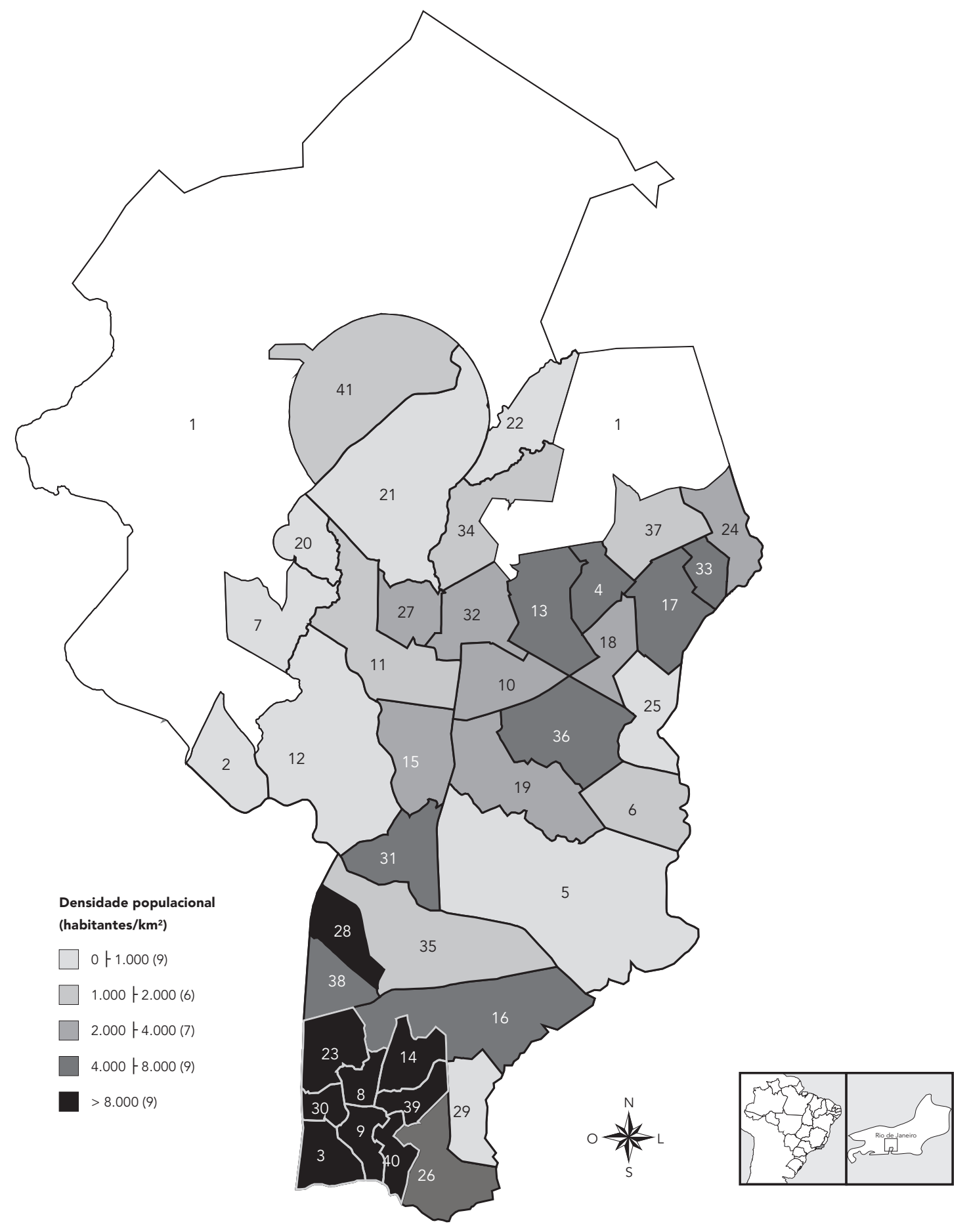

\begin{tabular}{ll}
0 & 2 \\
\hline
\end{tabular} 
Figura 3

Casos novos de hanseníase detectados em Duque de Caxias, Rio de Janeiro, Brasil, unidades Posto Médico Sanitário (PMS) e Programa Saúde da Família (PSF) com assistência à hanseníase e campanhas de rastreamento de casos da doença, por período.

a) Casos novos (1998-2000)

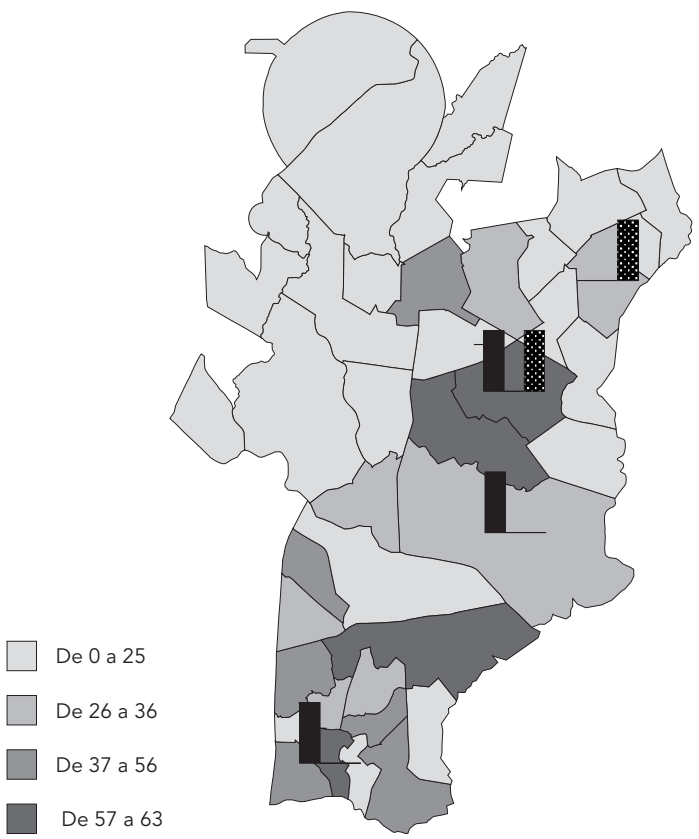

c) Casos novos (2004-2006)

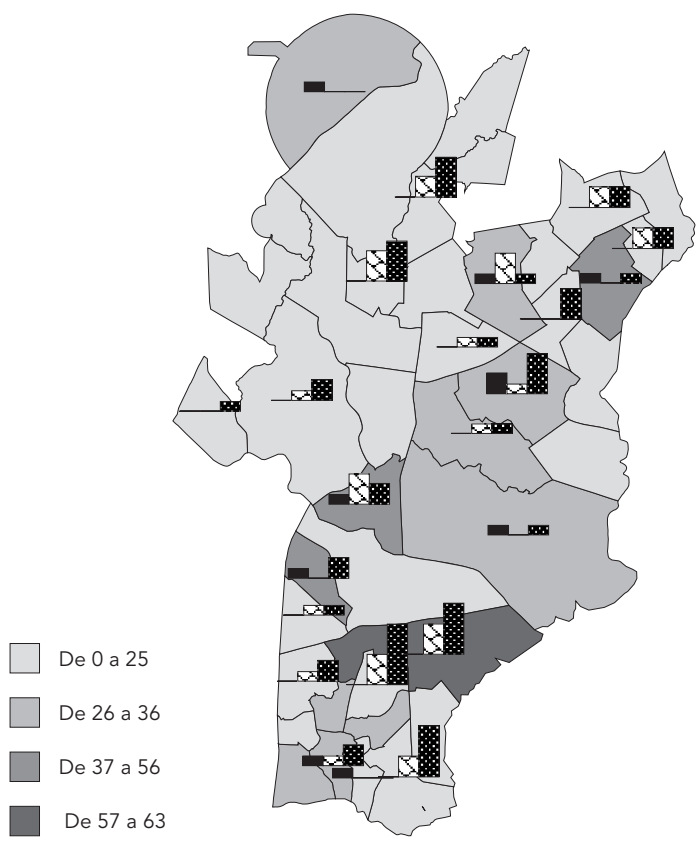

b) Casos novos (2001-2003)

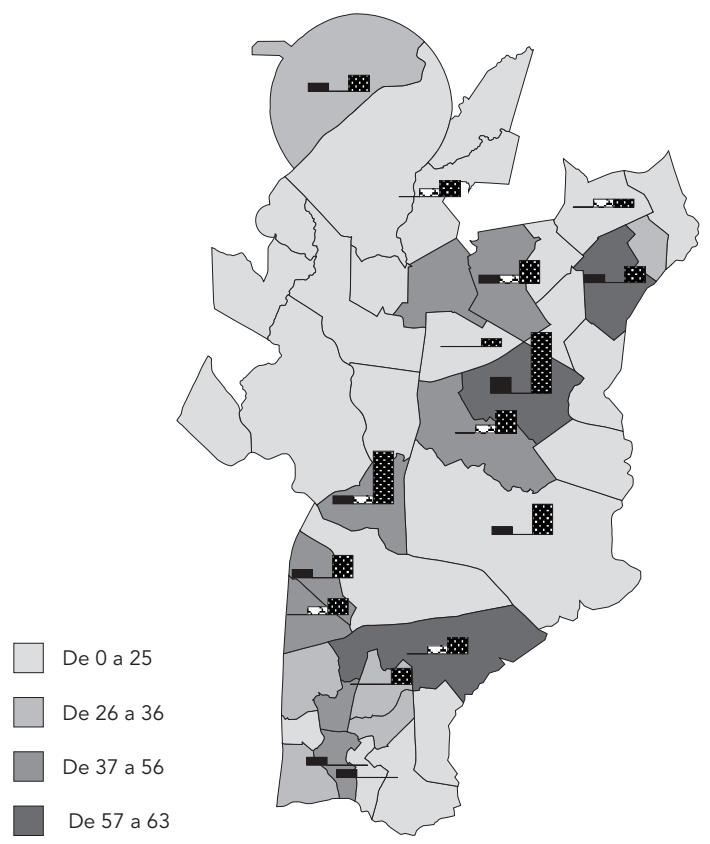

Gráfico de barras

Unidades PMS

E. Unidades PSF

d) Casos novos (1998-2006)

$\%$ Campanhas

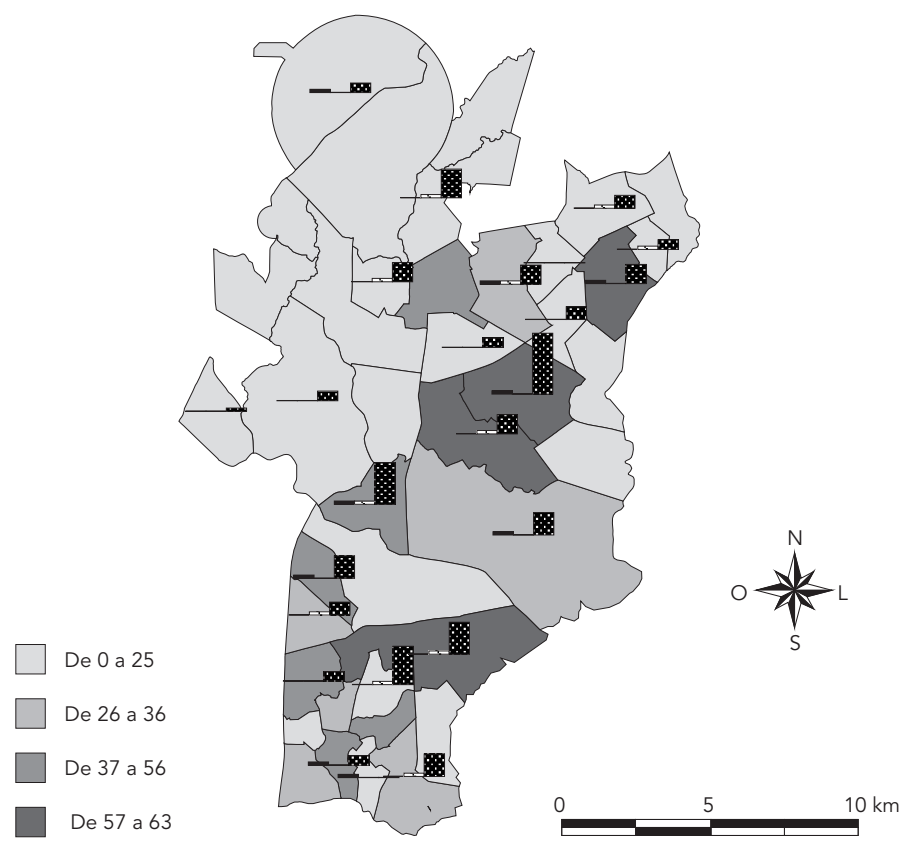


a) Taxa de detecção bruta

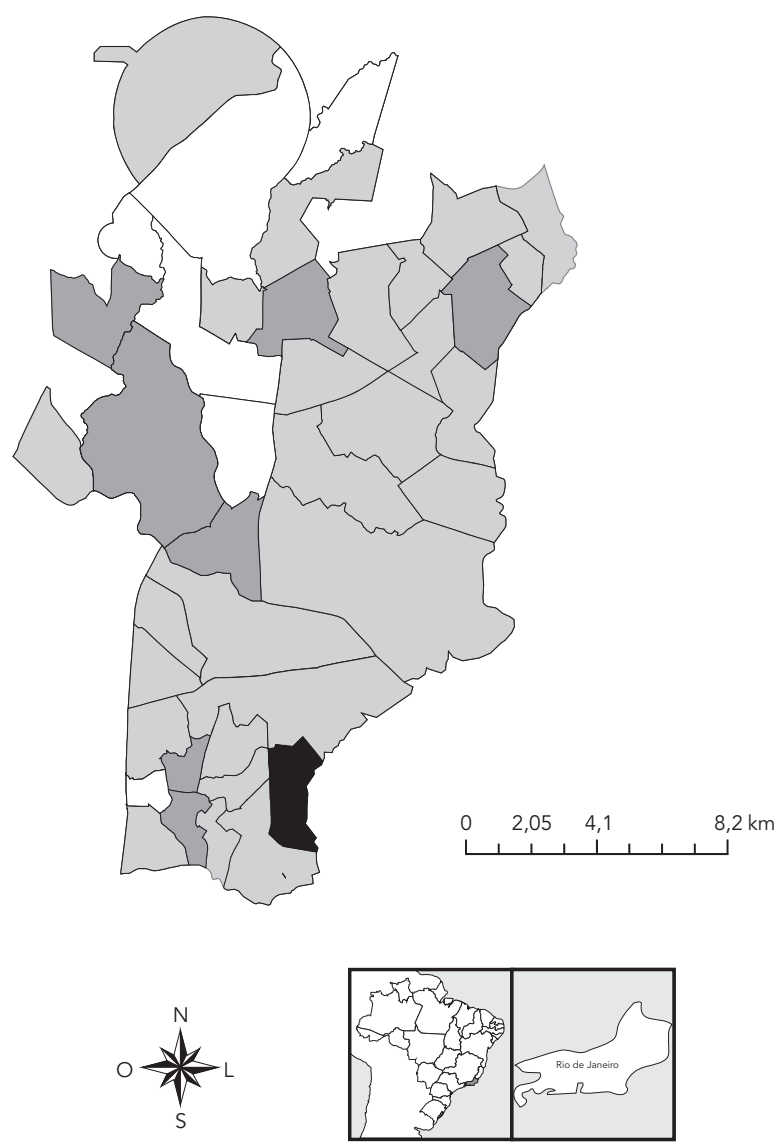

b) Taxa de detecção ajustada

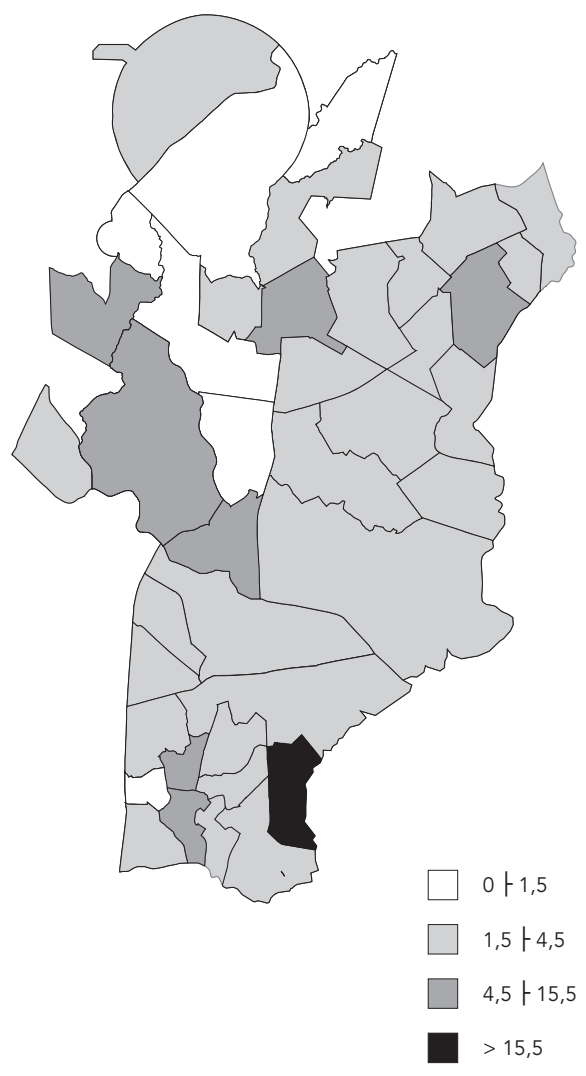

evolução bastante favorável e otimista do quadro epidemiológico ao longo dos nove anos. As ações de combate à doença, realizadas na região, refletiram-se em mudanças importantes do cenário endêmico e detecção mais precoce dos casos. Elevação e posterior redução da taxa de detecção foram vistas no período, associadas a uma redução dos casos em mulheres. Na literatura, não só há evidências de que o declínio da doença pode ser acompanhado de aumento na idade dos indivíduos no momento do diagnóstico, como também de que há maior proporção dos casos no sexo masculino e de formas multibacilares 20,21. Nesta análise, os casos novos detectados mostraram, ainda, tendência a uma maior proporção da forma indeterminada e menor proporção de casos já apresentando grau II de incapacidade física no momento do diagnóstico 22 . Esses dados são animadores, principalmente quando relacionados a uma doença crônica secular, cujo ciclo de transmissão ainda é pouco conhecido 4,10 . No entanto, ao observar espacialmente o problema, outras questões geográficas são levantadas, e um cenário novo se mostra menos otimista e com diferentes desafios.

Penna et al. 23, em 2009, realizaram análise da distribuição espacial da hanseníase no Brasil e verificaram aumento das taxas de detecção em algumas áreas (clusters) e, segundo os autores, isso poderia ser explicado pela melhoria do acesso aos serviços de assistência primária à saúde, ocorrido no mesmo período. No presente estudo, com a avaliação da distribuição espacial da frequência dos casos novos, foi possível demonstrar que as ações refletiram no aumento da detecção de casos novos nos bairros onde ocorreu maior 


\section{Figura 5}

Taxas de detecção dos casos novos de hanseníase, brutas e ajustadas (método bayesiano empírico local - LEB), em todo o período. Duque de Caxias, Rio de Janeiro, Brasil.

a) LISA MAP: taxa de detecção bruta

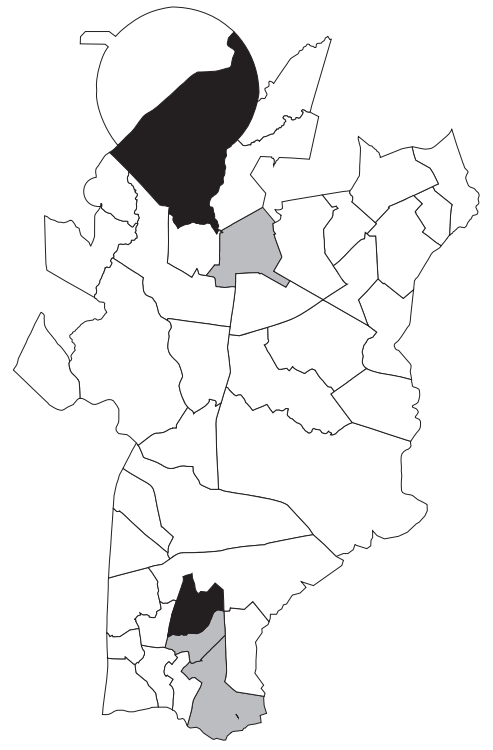

c) BoxMap: taxa de detecção bruta

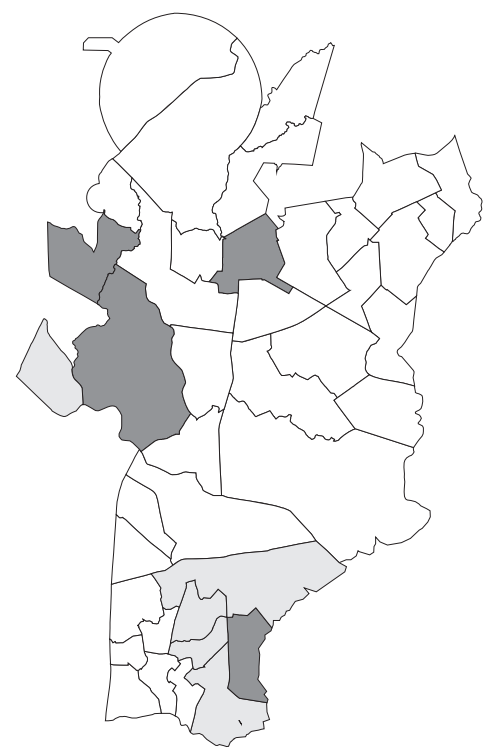

0

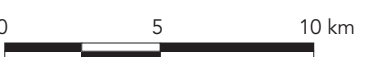

b) LISA MAP: taxa de detecção ajustada

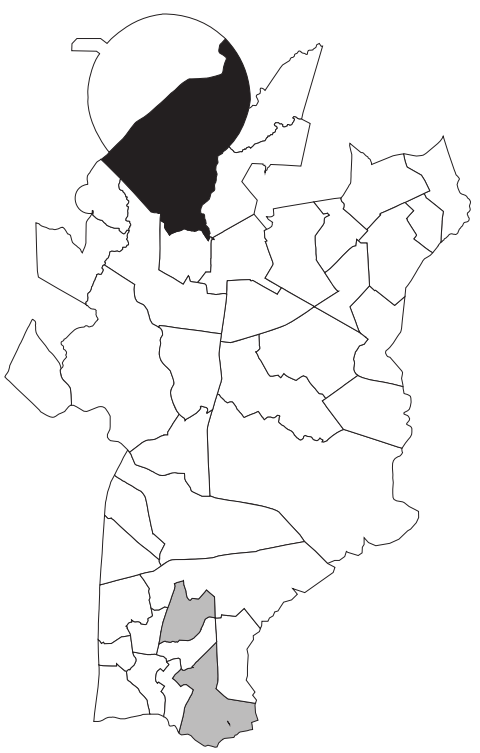

d) BoxMap: taxa de detecção ajustada

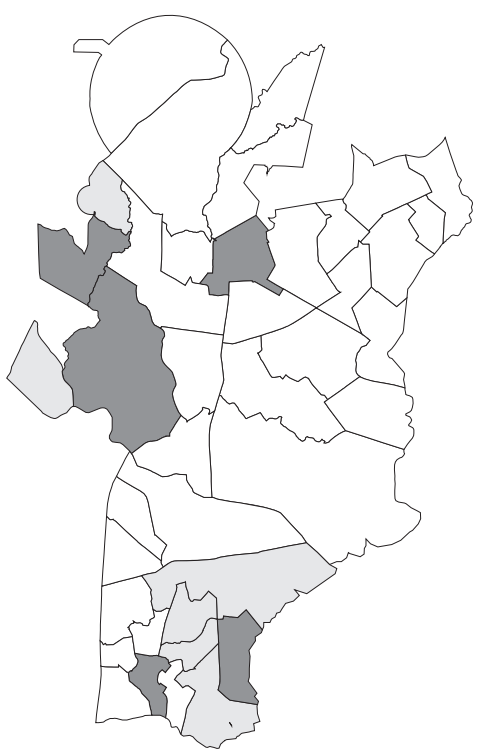

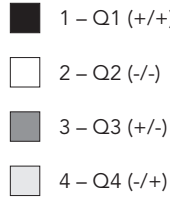

$0 \underbrace{N}_{S}$

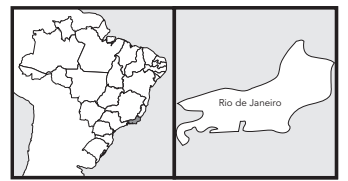


atuação do município. Mas, quando a avaliação é feita com as taxas de detecção, a distribuição espacial muda completamente de aspecto. A diferença espacial observada entre a frequência de casos e taxa parece mostrar dois processos distintos de um mesmo fato. As ações de rastreamento e de facilitação do acesso ao tratamento parecem agir de forma mais imediata numa das extremidades do problema: a consequência (o adoecimento); entretanto, na outra extremidade, com igual importância, encontram-se as causas (fatores de risco), que não estariam sendo atingidas por essas ações 24 .

Assim como a análise realizada por Lapa et al. 25, em 2001, em Olinda (Pernambuco), uma análise semelhante, na faixa sul-noroeste, referente à área de maiores taxas verificada em Duque de Caxias, poderia permitir compreensão melhor do quadro endêmico. Uma questão histórica relevante dessa área diz respeito ao início da colonização do município nas proximidades da Estrada Velha do Pilar, via que coincide, na maior parte de sua extensão, com a faixa de área crítica identificada na análise. Fatores regionais podem estar delimitando contextos específicos favorecedores da manutenção do bacilo circulante, tais como: ocupação do espaço urbano ou rural dessa região 25; características de moradia; hábitos de agregação familiar e social na dinâmica de inter-relação pessoal; fluxos de pessoas, motivados por economia ou cultura. Bastante útil será uma análise espacial descritiva ou, até mesmo, a regressão espacial utilizando esses possíveis fatores de risco para sua identificação, além de avaliar o peso e o papel de cada um na sustentação da infecção na região 26,27.

Outro enfoque do mesmo problema poderia ser a comparação genética entre cepas de amostras de pacientes residentes nas áreas com taxas de detecção com tendência de declínio e de ascensão, particularmente a faixa sul-noroeste identificada na análise espacial. Isso porque, quando indivíduos dentro de uma mesma comunidade são infectados por isolados idênticos, é possível que eles estejam ligados a uma cadeia recente de transmissão. Neste caso, a existência de portadores saudáveis, dentro da cadeia de transmissão, seria uma lacuna não alcançada pela poliquimioterapia. Por outro lado, quando os isolados são diferentes, há maior probabilidade de o fato estar relacionado a uma reativação de eventos antigos de transmissão, e não ligados à transmissão recente, como observado na reativação da tuberculose 28,29.

Com base em uma análise espacial da hanseníase no município, foi possível reconhecer subáreas mais delimitadas, com complexidade própria, que devem ser mais minuciosamente exploradas. Essa nova visão, aparentemente contrária aos dados gerais descritivos, reflete apenas as diferentes conclusões para uma mesma realidade, dependendo de como se olha para o contexto e em que escala.

A meta de eliminação da hanseníase como problema de saúde pública, de menos de um caso por 10 mil habitantes, foi definida pela OMS e seguida pelo Ministério da Saúde. Foi, ainda, pactuada entre as diretrizes do Pacto pela Vida, na reunião da Comissão Intergestores Tripartite do dia 26 de janeiro de 2006. Ressalta-se, desse modo, a importância do combate a essa doença causadora de grandes sofrimentos físicos e sociais.

As metas e objetivos do Pacto pela Vida estabelecem as responsabilidades sanitárias e atribuições do respectivo gestor, nos três níveis, assim como os indicadores de monitoramento, que integram os diversos processos de pactuação de indicadores existentes 30 . Outras metas que têm colaborado para a redução da endemia no país são a descentralização estendida a todas as unidades de saúde, para o diagnóstico precoce e o menor risco de desenvolvimento de incapacidades físicas, assim como a informação, educação e comunicação sobre a hanseníase, para tornar o indivíduo capaz de se autoproteger, identificando os sinais e sintomas da doença precocemente. Sabe-se, porém, que algumas regiões mantêm altas taxas de prevalência e detecção, chamadas de regiões prioritárias pelo Ministério de Saúde. Essas localidades possuem estruturas próprias que mantêm o bacilo circulante. Novas análises que possam identificar essas áreas e características dessas estruturas podem permitir um combate mais efetivo.

A análise espacial da distribuição da hanseníase no Município de Duque de Caxias identificou cluster na faixa sul-noroeste, não relacionado diretamente às ações de campanhas ou descentralização. Esse achado reforça a possibilidade de esse tipo de análise ser usado como uma ferramenta importante para identificação de áreas críticas da endemia e para avaliação do impacto das ações estratégias de combate à doença realizadas na região, incluindo os fatores de risco relacionados às condições sociais, econômicas e sanitárias dos indivíduos expostos. Isso poderia ampliar e corroborar com as já utilizadas, na identificação dos fatores perpetuadores da endemia. A partir disso, será possível, por um lado, planejar ações preventivas nas áreas identificadas de maior risco e, por outro lado, planejar intervenções estratégicas e monitoramento seletivo nessas áreas, de forma mais eficiente, fomentando, assim, maior impacto nas mudanças do quadro epidemiológico da região. 


\section{Resumo}

O objetivo foi identificar o padrão espacial da ocorrência da hanseníase em Duque de Caxias, município de alta endemicidade da doença no Estado do Rio de Janeiro, Brasil. Foram selecionados todos os casos novos de hanseníase registrados no banco do Sistema de Informação de Agravos de Notificação (SINAN), entre 1998 e 2006. Realizou-se análise por período subdividido a cada três anos, seguido de análise espacial por meio da estimativa bayesiana empírica local e do cálculo da autocorrelação espacial global (Moran) e local (LISA). A análise mostrou melhora acentuada do quadro epidemiológico, com o diagnóstico mais precoce. Houve redução da proporção de casos com grau II de $13,6 \%$ para $8,6 \%(p=0,04)$. Verificou-se aumento da detecção de casos com forma indeterminada, de 10,3\% para $18 \%(p=0,00)$. A análise espacial identificou cluster na faixa sul-noroeste, não relacionado diretamente às ações de campanhas ou descentralização, mostrando ser uma ferramenta importante para identificação de áreas críticas da endemia e para avaliação do impacto das ações estratégias de combate à doença.

Hanseníase; Análise Espacial; Doenças Endêmicas

\section{Colaboradores}

M. Duarte-Cunha e R. Souza-Santos participaram da concepção do projeto, análise e interpretação dos dados, redação e aprovação final da versão. H. J. Matos e M. L. W. Oliveira colaboraram na concepção do projeto e aprovação final da versão.

\section{Referências}

1. Lockwood DNJ, Suneetha S. Leprosy: too complex a disease for a simple elimination. Bull World Health Organ 2005; 83:230-5.

2. Sarno EN. Hansen's disease in the laboratory. Hist Ciênc Saúde-Manguinhos 2003; 10 Suppl 1: 277-90.
3. van Beers SM, de Wit MYL, Klatser PR. The epidemiology of Mycobacterium leprae: recent insight. FEMS Microbiol Lett 1996; 136:221-30.

4. Noordeen SK. The epidemiology of leprosy. In: Haastings RC, editor. Leprosy. Edinburgh: Churchil Livinstong; 1985. p. 15-30. 
5. Meima A, Smith WCS, Oortmarssen GJV, Richardus $\mathrm{JH}$, Habbema JD. The future incidence of leprosy: a scenario analysis. Bull World Health Organ 2004; 82:373-80.

6. Oskam L, Slim E, Buhrer-Sékula S. Serology: recent developments, strengths, limitations and prospects: a state of the art overview. Lepr Rev 2003; 74:196-205.

7. Queiroz JW, Dias GH, Nobre ML, Sousa Dias MC, Araújo SF, Barbosa JD, et al. Geographic information systems and applied spatial statistics are efficient tools to study Hansen's disease (leprosy) and to determine areas of greater risk of disease. Am J Trop Med Hyg 2010; 82:306-14.

8. Bakker MI, Hatta M, Kwenang A, Faber WR, van Beers SM, Klatser PR, et al. Population survey to determine risk factors for Mycobacterium leprae transmission and infection. Int J Epidemiol 2004; 33:1329-36.

9. Chatterjee BR, Taylor CE, Thomas J, Naidu GN. Acid fast bacillary positivity in asymptomatic individuals in leprosy endemic villages around Jhalda in West Bengal. Lepr India 1976; 48:119-31.

10. Moreira MBR, Penna GO, Pereira GFM, Madalena M. Guia para o controle da hanseníase. Brasília: Departamento de Atenção Básica, Secretaria de Políticas Públicas, Ministério da Saúde; 2002. (Cadernos de Atenção Básica, 10).

11. Aquino NM. Guia de vigilância epidemiológica. 6 a Ed. Brasília: Ministério da Saúde; 2005. (Série A. Normas e Manuais Técnicos).

12. Ignotti E. Abandonos ou abandonados? Aspectos da hanseníase no Município de Duque de Caxias, Rio de Janeiro [Dissertação de Mestrado]. Rio de Janeiro: Escola Nacional de Saúde Pública, Fundação Oswaldo Cruz; 1999.

13. Cunha MD. O impacto das estratégias de eliminação da hanseníase em município endêmico do Estado do Rio de Janeiro, Brasil [Dissertação de Mestrado]. Rio de Janeiro: Faculdade de Ciências Médicas, Universidade do Estado do Rio de Janeiro; 2005.

14. Secretaria Municipal de Saúde de Duque de Caxias. Relatório anual. Duque de Caxias: Coordenadoria de Saúde Coletiva, Secretaria Municipal de Saúde de Duque de Caxias; 2007.

15. Laguardia J, Domingues CMA, Carvalho C, Lauerman CR, Macário E, Glatt R. Sistema de informação de agravos de notificação em saúde (Sinan): desafios no desenvolvimento de um sistema de informação em saúde. Epidemiol Serv Saúde 2004; 13:135-46.

16. Rodrigues AF, Escobar AL, Souza-Santos R. Análise espacial e determinação de áreas para controle da malária no Estado de Rondônia. Rev Soc Bras Med Trop 2008; 41:55-64.
17. Ximenes RAA, Martelli CMT, Souza WV, Lapa TM, Albuquerque MFM, Andrade ALSS, et al. Vigilância de doenças endêmicas em áreas urbanas: a interface entre mapas de setores censitários e indicadores de morbidade. Cad Saúde Pública 1999; 15:53-61.

18. Souza-Santos R, Carvalho MS. Análise da distribuição espacial de larvas de Aedes aegypti na Ilha do Governador, Rio de Janeiro, Brasil. Cad Saúde Pública 2000; 16:31-42.

19. Santos SM, Souza WV. Introdução à estatística espacial para a saúde pública. Brasília: Ministério da Saúde/Fundação Oswaldo Cruz; 2007. (Série Capacitação e Atualização em Geoprocessamento em Saúde, 3).

20. Meima A, Irgens LM, Oortmarssen GJV, Richardus JH, Habbema JD. Disappearance of leprosy from Norway: an exploration of critical factors using an epidemiological modeling approach. Int J Epidemiol 2002; 31:991-1000.

21. Irgens LM, Skjaerven R. Secular trends in age at onset, sex ratio, and type index in leprosy observed during declining incidence rates. Am J Epidemiol 1985; 122:695-705.

22. Ridley DS, Jopling WH. Classification of leprosy according to immunity. A five-group system. Int J Lepr Other Mycobact Dis 1966; 34:255-73.

23. Penna ML, Oliveira ML, Penna GO. The epidemiological behaviour of leprosy in Brazil. Lepr Rev 2009; 80:332-44.

24. Amaral EP, Lana FC. Análise espacial da hanseníase na microrregião de Almenara, MG, Brasil. Rev Bras Enferm 2008; 61(n.spe):701-7.

25. Lapa T, Ximenes R, Silva NN, Souza W, Albuquerque MFM, Campozana G. Vigilância da hanseníase em Olinda, Brasil, utilizando técnicas de análise espacial. Cad Saúde Pública 2001; 17:1153-62.

26. Imbiriba EN, Silva Neto AL, Souza WS, Pedrosa V, Cunha MG, Garnelo L. Social inequality, urban growth and leprosy in Manaus: a spatial approach. Rev Saúde Pública 2009; 43:656-65.

27. Silva DR, Ignotti E, Souza-Santos R, Hacon SS. Hanseníase, condições sociais e desmatamento na Amazônia brasileira. Rev Panam Salud Pública 2010; 27:268-75.

28. Matsuoka M, Maeda S, Kai M, Nakata N, Chae GT, Gillis TP, et al. Mycobacterium leprae typing by genomic diversity and global distribution of genotypes. Int J Lepr Other Mycobact Dis 2000; 68:121-8.

29. Young D. Prospects for molecular epidemiology of leprosy. Lepr Rev 2003; 74:11-7.

30. Ministério da Saúde. Diretrizes operacionais dos Pactos pela Vida, em defesa do SUS e de gestão. Brasília: Ministério da Saúde; 2006.

Recebido em 06/Jul/2011

Versão final reapresentada em 22/Dez/2011

Aprovado em 16/Jan/2012 